\title{
Self-Triggered Control over Wireless Sensor and Actuator Networks
}

\author{
José Araújo*, Adolfo Anta ${ }^{\dagger}$, Manuel Mazo Jr..$^{\ddagger}$, João Faria*, \\ Aitor Hernandez*, Paulo Tabuada ${ }^{\S}$ and Karl H. Johansson* \\ *ACCESS Linnaeus Center, Royal Institute of Technology, Stockholm, Sweden \\ Email: \{araujo,jfff,aitorhh,kallej\}@ee.kth.se \\ ${ }^{\dagger}$ TU Berlin \& Max Planck Institute, Germany \\ Email: anta@control.tu-berlin.de \\ ${ }^{\ddagger}$ INCAS $^{3}$ \& University of Groningen, The Netherlands \\ Email: m.mazo@ rug.nl \\ §University of California at Los Angeles, USA \\ Email: tabuada@ee.ucla.edu
}

\begin{abstract}
Energy and communication bandwidth are scarce resources in wireless sensor and actuator networks. Recent research efforts considered the control of physical processes over such resource limited networks. Most of the existing literature addressing this topic is dedicated to periodically sampled control loops and scheduled communication, because it simplifies the analysis and the implementation. We propose instead an aperiodic network transmission scheme that reduces the number of transmission instances for the sensor and control nodes, thereby reducing energy consumption and increasing network lifetime, without sacrificing control performance. As an added benefit, we show the possibility of dynamically allocating the network bandwidth based on the physical system state and the available resources. In order to allow timely, reliable, and energy efficient communication, we propose a new co-design framework for the wireless medium access control, compatible with the IEEE 802.15.4 standard. Furthermore, we validate our approach in a real wireless networked control implementation.
\end{abstract}

\section{INTRODUCTION}

Increasingly, control systems are operated over large-scale, networked infrastructures. The use of wireless communication technology provides major advantages in terms of increased flexibility, and reduced installation and maintenance costs. Following this trend, several vendors are introducing devices that communicate over low-power wireless sensor networks (WSNs) for industrial automation and process control. While WSNs have been widely analyzed and deployed to extract information from the physical world [1], actuation over wireless networks is still taking its first steps. In this paper we focus on the use of wireless sensors and actuator networks to control large-scale physical systems. Examples of such systems are irrigation networks of open water channels [2], where water flow is regulated by gates along the channels, or energy efficient buildings [3], where sensors are deployed to

The work of the authors was supported by the ${ }^{*}$ Knut and Alice Wallenberg Foundation, the ${ }^{*}$ Swedish Research Council, the ${ }^{*}$ Swedish Strategic Research Foundation and the ${ }^{*}$ Swedish Governmental Agency for Innovation Systems,

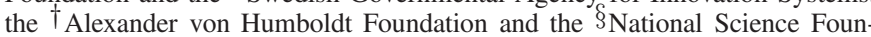
dation award 0834771 adjust heating, ventilation and air conditioning (HVAC) and illumination. In particular, our main concern is to efficiently design and implement such control systems to extend the life span of the wireless nodes.

An extensive set of tools and techniques have been developed to reduce power consumption in WSNs. Unfortunately, the situation is much less favorable for wireless sensor and actuator networks (WSANs). While much work has been devoted by the controls community to the study of wired networked control systems, not so much attention has been devoted to the specific problems arising in the wireless context. Traditional control engineering does not consider implementation requirements such as the minimization of communication between sensors, controllers and actuators. Such minimization in a large-scale wireless context is crucial both for energy savings and bandwidth reduction. Existing studies on this topic either neglect the dynamics of the physical system [4], [5] or do not provide guarantees on the stability of the physical systems being controlled [6]. In particular, most efforts of the network control systems community have been conducted under the assumption of periodic sampling and actuation [7], which, in general, may require data rates not practical in a wireless system. To address these issues, two new control paradigms have recently been proposed to efficiently use the communication resources in an aperiodic manner while enforcing pre-specified control performance: event-triggered control [8], [9], [10], [11] monitors the state of the plant to select the time instants at which the control input needs to be updated; while self-triggered control [12], [13], [14], [15] represents a model-based emulation of event-triggered control. Self-triggered control presents some advantages over event-triggered control by removing the need for continuous monitoring of the plant and by providing transmission times in advance to allow for efficient scheduling. Moreover, both selftriggered and event-triggered control strategies are techniques applicable to a large set of systems (including nonlinear), provide guarantees on the control performance (in the form of 


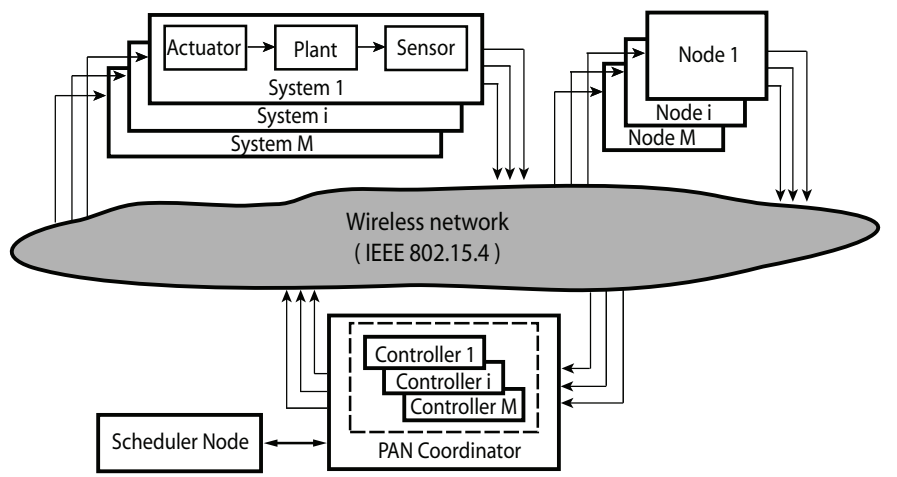

Fig. 1: Wireless Sensor and Actuator Network System Topology. Several control loops and independent sensors share information over the same low power wireless IEEE 802.15.4 network.

rates of convergence) and can accommodate bounded delays. To the best of our knowledge, the only existing implementation of self-triggered control appeared in [16], where the control of a double integrator over a wired Controller Area Network $(\mathrm{CAN})$ is considered. We present in this paper the first wireless implementation of self-triggered control. The wireless nature of the communication medium renders scheduling and energy considerations much more critical, and poses a set of new problems with respect to wired control. Hence, the present work represents a major step towards the design of resourceaware implementations of control laws over WSANs. We demonstrate the applicability of self-triggered control of a physical system over a WSAN and its advantages with respect to the traditional periodic paradigm, both in terms of energy savings and bandwidth reduction. Our main contribution is the proposal and use of a modification of the low-power wireless IEEE 802.15.4 protocol [17] for self-triggered control implementations. Moreover, we propose the scheduling of messages according to an Earliest-Deadline-First (EDF) policy, and present necessary and sufficient conditions for schedulability. We have implemented the proposed design on a physical system composed of two coupled water tanks and several monitoring nodes. The experimental results presented in this paper show that for the considered physical system, protocol and scheduling policy, the self-triggered strategy reduces battery consumption in the sensor and actuator nodes by $41 \%$ with respect to a conventional periodic strategy.

The rest of the paper is organized as follows. We introduce the problems we address in Section II. Section III revisits the self-triggered paradigm and Section IV describes the IEEE 802.15.4 protocol and the proposed modifications to the standard. Section V describes the experimental setup and the hardware used and Section VI presents the results and energy measurements. Finally, Section VII summarizes the main ideas and proposes possible extensions of this work.

\section{Control OVEr Wireless Networked Systems}

The problem we aim to solve is that of performing control over a potentially large and complex wireless networked system. Given a control loop that is closed over a wireless network, we are interested in solutions that guarantee stability and performance of the control system while minimizing the energy consumption of the wireless sensor and actuator nodes involved. An example of such a networked system is depicted in Fig. 1, where several control systems and other independent nodes are coordinated and scheduled by a Personal Area Network (PAN) coordinator.

We consider control systems of the form:

$$
\dot{\mathrm{x}}=f(\mathrm{x}, \mathrm{u}), \quad \mathrm{x}(t) \in \mathrm{R}^{n}, \mathrm{u}(t) \in \mathrm{R}^{m}
$$

for which a feedback controller:

$$
\mathrm{u}=k(\mathrm{x})
$$

is designed to render the closed-loop system $\dot{\mathrm{x}}=f(\mathrm{x}, \mathrm{u})$ asymptotically stable. We denote the state trajectory of the control system by $\mathrm{x}$ and the input trajectory by $\mathrm{u}$.

The controller (2) is implemented on the PAN coordinator which communicates through the wireless network with the sensors and actuators connected to the plant, sharing the same network with other independent nodes as presented in Fig. 1. Whenever there is the need of a control update, the wireless sensors connected to the plant sample the state $x$ at time instant $t_{i}$ and send the messages to the controller (sensor messages). Upon the arrival of this message, the PAN coordinator computes the control input $\mathrm{u}=k\left(\mathrm{x}\left(t_{i}\right)\right)$. When the computation is finished, the PAN coordinator sends a message to the wireless actuators with the new input $u$, which is applied to the plant (actuator message) at time $t_{i}+\delta$ where $\delta$ corresponds to the delay between measurement and actuation. We assume that the actuators hold the previous control input value until a new message from the controller is received. The network is shared between the sensors, controllers, actuators and other independent nodes which might transmit information over the network in a periodic or aperiodic fashion.

The problems we solve in this paper are the following:

- When should the sensor and actuator messages be sent over a wireless network, so that stability is guaranteed and desired performance is achieved, while keeping energy consumption at the nodes low?

- Is it possible to design and implement, with off-theshelf technology, a WSAN that would solve the preceding problem?

In order to address these issues, we first revisit a control paradigm that leads to a more efficient utilization of the available resources.

\section{Self-Triggered Control}

For simplicity, we focus our exposition on physical systems that can be described by linear differential equations, although similar ideas can be developed for systems described by nonlinear differential equations [13]. We consider linear control systems of the form:

$$
\dot{\mathrm{x}}=A \mathrm{x}+B \mathrm{u}, \quad \mathrm{x}(t) \in \mathbb{R}^{n}, \mathrm{u}(t) \in \mathbb{R}^{m}
$$


where $A$ and $B$ are matrices of appropriate dimensions. A controller $\mathrm{u}=K \mathrm{x}$ is usually designed to render the system asymptotically stable and $u$ remains constant between two consecutive control updates. Hence there exists a Lyapunov function of the form $V=\mathrm{x}^{T} P \mathrm{x}$ satisfying

$$
\dot{V}=\frac{\partial V}{\partial \mathrm{x}}(A+B K) \mathrm{x}=-\mathrm{x}^{T} Q \mathrm{x}
$$

where $Q$ is a positive definite matrix. The Lyapunov function $V$ can be seen as a certificate of stability, since according to equation (4) $V$ is always decreasing, but also of performance since (4) also ensures that the rate of decrease is at least $\mathrm{x}^{T} Q \mathrm{x}$. As mentioned in the previous section, the input $u$ cannot be updated continuously but only at discrete time instants $t_{i}$, whenever an actuator message is received. Traditionally, control-related messages are exchanged periodically, that is, $t_{i+1}-t_{i}=T$ for all $i \in \mathbb{N}_{0}$. The period $T$ is chosen in order to guarantee stability and desired performance under all possible operating conditions. This approach represents a conservative solution to the message scheduling problem since $T$ is selected based on a worst-case scenario. On the other hand, self-triggered control considers the plant model and the current state of the system to decide the next transmission time for the sensor and actuator messages.

Since the input $\mathrm{u}$ is kept constant between updates, the evolution of the Lyapunov function $V$ for the implementation is now given by:

$$
\dot{V}=\frac{\partial V}{\partial \mathrm{x}}\left(A \mathrm{x}+B K \mathrm{x}\left(t_{i}\right)\right)
$$

As the evolution of $V$ determines the behavior of the system, we specify the desired performance for the implementation by means of a function $S$ upper bounding the evolution of $V$ :

$$
V(t) \leq S(t) .
$$

The previous inequality guarantees that $V$ decreases at least as fast as $S$ does. In that sense we can regard $S$ as defining the control performance that the self-triggered implementation will guarantee. Among other options, a possible choice for $S$ is the Lyapunov function:

$$
S(t)=\mathrm{x}_{s}(t)^{T} P \mathrm{x}_{s}(t)
$$

for the hybrid system:

$$
\begin{aligned}
\dot{\mathrm{x}}_{s} & \left.=A_{s} \mathrm{x}_{s} \quad t \in\right] t_{i}, t_{i+1}[ \\
\mathrm{x}_{s}\left(t_{i}\right) & =\mathrm{x}\left(t_{i}\right)
\end{aligned}
$$

where $A_{s}$ is a Hurwitz matrix satisfying the following Lyapunov equation $A_{s}^{T} P+P A_{s}=-R$. The matrix $R$ has to be chosen so that $Q-R$ is positive definite, in order to guarantee a minimum inter-transmission time for the control messages (see [15] for details). In other words, the Lyapunov function of the implemented system (3) decays at least as fast as the Lyapunov function of the reference system (8).

Inequality (6) can be enforced by closing the loop whenever:

$$
V(t)=S(t)
$$

Indeed, notice that at every time instant $t_{i}$ we have $\dot{V}=-\mathrm{x}^{T}\left(t_{i}\right) Q \mathrm{x}\left(t_{i}\right)<\dot{S}=-\mathrm{x}^{T}\left(t_{i}\right) R \mathrm{x}\left(t_{i}\right)$ (since $Q-R$ is positive definite), and therefore $V(t)<S(t)$ for $t \in] t_{i}, t_{i+1}$. Equality (10) implicitly defines a sequence of time instants $t_{i}$ at which the input needs to be updated. This approach, known as event-triggered control, is expected to outperform the traditional periodic approach, since the current state of the system is considered to decide the next time at which messages need to be exchanged between controllers, sensors and actuators.

Self-triggered control represents a model-based emulation of event-triggered control. Self-triggered implementations identify the time instants $t_{i}$ at which (10) is satisfied, taking into account the plant model given by (3), the last measurement of the state of the system $\mathrm{x}\left(t_{i}\right)$ and the performance specification in (7). The prediction of the time between two consecutive updates is embodied in the function:

$$
\tau_{i}=t_{i+1}-t_{i}=g\left(\mathrm{x}\left(t_{i}\right), S\right) .
$$

Throughout the paper we will refer to $\tau_{i}$ as the intertransmission time. There exists several methods in the literature to compute such a function $g$ [14], [13], [15]. We focus on the technique developed in [15], although similar analyses can be carried out for the other available self-triggering techniques, including those for nonlinear systems. Due to space constraints we do not explain here how such formula is obtained, and we refer the interested reader to [15].

Notice that $t_{i+1}$ represents the time at which the input needs to be updated, therefore both a sensor message and an actuator message need to be delivered between $t_{i}$ and $t_{i+1}$. Since (11) defines the sequence of inter-transmission times, a scheduling analysis can be carried out beforehand to guarantee the schedulability of the control-related messages (see Section IV-C). In the next section we introduce the wireless network Medium Access Control (MAC) and scheduling designs, and the required schedulability analysis that guarantees a feasible self-triggered implementation of several control systems on a shared wireless network.

\section{WiRELESS COMMUNiCATIONS}

The standardization of low data rate and low power wireless networks is an ongoing process and there is not yet any widely accepted complete protocol stack, particularly for control [1]. The IEEE 802.15.4 protocol [17], which specifies physical and MAC layers, is the base of solutions in industrial automation such as WirelessHART and ISA100 [1]. These standards rely on a completely centralized TDMA scheduling approach for mesh networks, where dynamical schedule changes, required in event-based control and aperiodic sensor sampling has not been taken into account. Dynamic scheduling is part of the IEEE 802.15.4 standard MAC and a main interest of the IEEE 802.15.4e task group which works on MAC enhancements for process control and factory automation.

We are interested in implementing self-triggered control strategies in a large and heterogeneous, star topology WSAN. We also envision that other devices besides those involved 
in the control loops are present, sharing the same network resources. In what follows we consider the IEEE 802.15.4 protocol as the reference standard for our setup. Our main contribution comes in the form of suggested modifications in the standard MAC layer, and an implementation of these, to allow the implementation of feedback control under the selftriggered control paradigm.

\section{A. IEEE 802.15.4-based MAC}

The IEEE 802.15.4 standard specifies two types of medium access mechanisms depending on whether the network is in the beacon-enabled or the non beacon-enabled mode. Here we will focus in the beacon-enabled mode. In such a setup, a centralized coordinator node, the PAN coordinator, is responsible for synchronizing and configuring all the nodes in the network. The network topology in our study is a star network with all the messages going through the PAN coordinator where the controllers are located, as in Fig. 1. This specialized node is assumed to be connected to a power supply with a large amount of energy. The synchronization and configuration messages take place periodically at each beacon message which defines the time bounds of the superframe structure defined by the protocol. In what follows we denote by $\Gamma_{i}$ the time instants at which this beacon is transmitted. The superframe length is named Beacon Interval (B.I.) and is further divided in active and inactive periods, as shown in Fig. 2. The active period has a time interval defined by Superframe Duration (S.D.) and is divided in 16 equally sized slots. The active period is further split into a Contention Access Period (CAP) and a Collision Free Period (CFP). During the CAP, the MAC scheme is Carrier Sense Multiple Access/Collision Avoidance (CSMA/CA) where the nodes in the network sense if the channel is busy before transmitting a message. The CAP period is used by nodes to send best effort messages where packet drops can happen due to collision or channel congestion. On the other hand, the CFP is intended to provide real-time guaranteed service, by allocating Guaranteed Time Slots (GTS) to the nodes using it, in a Time Division Multiple Access (TDMA) scheme. Since during the CFP there are no packet losses due to collisions or channel congestion ${ }^{1}$, this mechanism is an attractive feature for time-sensitive wireless applications, as is the case of realtime control of several plants over a wireless network. The total number of GTS slots is limited to 7 in the current standard. We respect this limitation in the present study as it does not pose a limitation for our application. However, this value could be easily changed if required. Additionally, during the active period an acknowledgement mechanism is present: nodes receive a short acknowledgement packet after each transmission indicating that its packet was received. Since this standard is designed for low-power applications, an inactive period is defined in the end of the active period so the network nodes and the PAN coordinator enter a low-power mode and

\footnotetext{
${ }^{1}$ We neglect wireless interference since we assume that there are no external nodes in the network using the same frequency bands.
}

TABLE I: Current consumption of a Telos wireless platform.

\begin{tabular}{|c|l|l|}
\hline Mode & Description & Measure Current \\
\hline 1 & $\mu \mathrm{C}$ active, Radio Tx & $21.7 \mathrm{~mA}$ \\
\hline 2 & $\mu \mathrm{C}$ active, Radio Rx/listen & $22.8 \mathrm{~mA}$ \\
\hline 3 & $\mu \mathrm{C}$ active, Radio OFF & $2.4 \mathrm{~mA}$ \\
\hline 4 & $\mu \mathrm{C}$ idle, Radio OFF & $40 \mu \mathrm{A}$ \\
\hline
\end{tabular}

save energy. After this period, all the nodes leave the lowpower mode in order to receive the beacon message. In order to save energy in a wireless networked control system, it is necessary to understand how the current is consumed in the different radio and microcontroller $(\mu \mathrm{C})$ modes according to the specified protocol.

1) Power Consumption: The power consumption of the widely used wireless sensor platforms Telos [18] is given in Table I [19]. These platforms are equipped with the TI MSP430F1611 $\mu \mathrm{C}$ and the CC2420 radio, IEEE 802.15.4 compliant. The table shows the amount of current needed in different modes by the wireless platform according to radio and $\mu \mathrm{C}$ usage. These values show that it is highly power demanding to send and receive messages, and that receiving and listening requires more current than sending. Naturally, to save large amounts of energy the nodes should be in mode 4 for most of the time, achieved with large B.I. values for a fixed S.D., and reducing the amount of listening time and receptions/transmissions (mode 1 and 2). To be able to enlarge the B.I. value, it is crucial that the self-triggered control design from Sec. III stabilizes the system with a large minimum intertransmission time (11). Likewise, the triggering rule (6) should generate the least amount of samples possible, since each trigger generates a sequence of sensor transmissions, controller receptions/transmissions and actuator receptions. Following these considerations we are now ready to propose the protocol design approach to suit the self-triggered control paradigm.

2) MAC Design for Self-Triggered Control: The difference between our MAC and the specification in the IEEE 802.15.4 standard lies in the GTS allocation mechanism. We propose the use of a centralized scheduler node connected to the PAN coordinator for the assignment of GTS slots instead of the firstcome-first-served (FCFS) request-based scheme in the IEEE 802.15.4 MAC specification. The used scheduling algorithm is presented in Section IV-B. The scheduler node is responsible for, according to the selected scheduling algorithm, informing the PAN coordinator of which GTS is assigned to which specific node in the next superframe. At each superframe, all the network nodes have the radios ON to receive the beacon message from the PAN coordinator. This beacon contains the information necessary to configure the nodes that have an allocated GTS and inform them of the B.I and S.D. values. If a node has a GTS allocated, then it will transmit during that time and otherwise it will enter in the low-power mode during the B.I..Let us denote the minimum inter-transmission time for both the sensor and actuator messages as $t_{\text {min }}$ for all the possible plant conditions. This time will be formally defined in Sec. IV-C and it always guarantees a certain level of performance and stability of the closed loop system as 


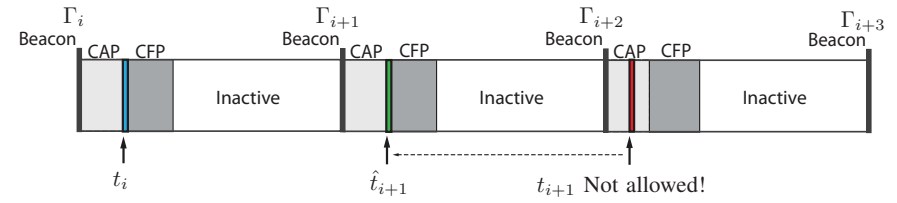

Fig. 2: EDF Scheduling over WSANs. Here we describe the scheduling of one of the nodes in the network using the selftriggered control scheme. The required triggering time $t_{i+1}$ is not allowed since it does not occur during a CFP. An adjustment of this time to $\hat{t}_{i+1}$ is made in order to allocate $t_{i+1}$ inside a GTS and guarantee (6) under EDF scheduling.

discussed in Sec. III. When defining the B.I. one needs to enforce B.I. $<t_{\min }$ to guarantee a desired level of performance and stability according to (6). This means that every time a node transmits a sample, one needs to ensure that it can do it again in at least $t_{\min }$. When a B.I. is chosen, a superframe duration S.D. $\leq$ B.I. follows.

\section{B. GTS Scheduling over WSANs}

As proposed in Sec. IV-A a scheduler node is responsible for the scheduling of GTS slots for the nodes in the network. This node is connected to the PAN coordinator, having access to all the information transmitted by the sensor nodes. In order to allow for efficient usage of the available network resources, we propose to schedule the messages in the network according to an Earliest Deadline First (EDF) approach, which is know to be optimal for time-constrained schedules [20]. In what follows we denote by $\triangle \mathrm{CAP}$ and $\triangle \mathrm{CFP}$ the values of the CAP and CFP duration, respectively. The design of the GTS scheduling should take into account the following facts:

1) There are two types of messages: hard messages with high priority and hard deadlines, and soft messages with lower priority. The scheduling of both hard and soft messages should be done according to independent EDF schemes.

2) The GTS scheduling algorithm should only schedule the triggering times $t_{i+1}$ given by (11), when $t_{i+1} \in\left[\Gamma_{i}+\right.$ $\left.\Delta \mathrm{CAP}, \Gamma_{i+1}+\Delta \mathrm{CAP}\right]$. If $t_{i+1}>\Gamma_{i+1}+\Delta \mathrm{CAP}$, then the scheduler will only assign a GTS slot to the requesting node in a later superframe. $\Gamma_{i}$ denotes the superframe $i$ start time.

3) The triggering times $t_{i+1}$ need to be adjusted to new values $\hat{t}_{i+1} \leq t_{i+1}$ if $\hat{t}_{i+1} \leq \Gamma_{i+1}+\Delta \mathrm{CAP}$ in order to fit the triggering time inside the GTS.

This last condition is illustrated in Fig. 2, where the triggering time $t_{i+1}$ is adjusted to be $\hat{t}_{i+1} \leq t_{i+1}$ since $\hat{t}_{i+1} \leq \Gamma_{i+1}+\Delta$ CAP, to prevent the transmission from falling in the CFP time two superframes ahead.

\section{Offline Schedulability Analysis}

In this section we analyze the schedulability of a set of hard messages under the IEEE 802.15.4 protocol. As mentioned before, the active period is divided into the CAP and the CFP. Since no guarantees can be provided during the CAP, we assume that all hard messages are sent during the CFP. During this window, messages are scheduled according to the EDF algorithm (see previous section).

Each message can be characterized by a triple $\left(t_{\min }, C, d\right)$, where $t_{\min }$ represents the period of a message (or minimum inter-transmission time for aperiodic messages), $C$ is the maximum transmission time and $d$ is the relative deadline (not necessarily equal to the period $t_{\min }$ ). Notice that control loops involve at least two types of messages: sensor to controller and controller to actuator. Sensor messages are always followed by actuator messages, hence they are never sent at the same time. To model this precedence constraint, we assume an offset $\phi_{a}$ for the actuator messages, equal to the deadline of the sensor message plus the computation time of the control law at the control node.

The inter-transmission times of a pair sensor-actuator message is defined by equation (11). Since offline the scheduler is not aware of the evolution of the state, worst-case inter-transmission times need to be considered in the offline schedulability analysis. The minimum inter-transmission time for both the sensor and the actuator message is given by $t_{\text {min }}=\min _{i} t_{i}$. However, since the transmission times $t_{i}$ depend on state of the plant and are not known in advance, we need to select $t_{\min }$ as the worst inter-transmission time over all possible initial conditions in the operating region $\Omega$ :

$$
t_{\text {min }}=\min _{x_{0}} g\left(x_{0}, S\right), \quad x_{0} \in \Omega
$$

Indeed, as in the case of periodic implementations, enough resources need to be reserved beforehand assuming worst-case conditions, even though these might rarely occur. However, as the state of the plant is measured the self-triggered policy modifies these requirements in run-time and reserved bandwidth can be reallocated among existing nodes. This property represents the main advantage of the self-triggered paradigm.

The deadline of the actuator message represents the maximum admissible bound on the delay between a sensor message is received by the controller and the arrival of the actuator message. The deadline of the sensor message represents the maximum admissible bound between the measurement of a sensor and the arrival of its corresponding sensor message. Notice that for control systems only the delay $\delta$ between measurement and actuation is relevant, i.e., the sum of the sensor and actuator deadlines.

Given a set of $n$ hard messages plus $p$ control loops, the schedulability conditions (sufficient and necessary) under nonpreemptive EDF are [21]:

$$
\begin{aligned}
\sum_{i=1}^{n+2 p} \frac{C_{i}}{t_{\min i}} & \leq 1 \\
\sum_{i=1}^{n+2 p}\left\lceil\frac{t-d_{i}-\phi_{i}}{t_{\min i}}\right\rceil^{+} C_{i}+C_{m} & \leq t, \quad \forall t \in S
\end{aligned}
$$

where the set $S$ is defined as:

$$
\begin{aligned}
S & =\cup_{i=1}^{n+2 p} S_{i}, \\
S_{i} & =\left\{d_{i}+m t_{\min i}: m=0,1, \ldots,\left\lfloor\frac{t_{\max }-d_{i}-\phi_{i}}{t_{\min i}}\right\rfloor\right\},
\end{aligned}
$$




$$
\begin{aligned}
t_{\max }= & \max \left\{d_{1}, \ldots, d_{n+2 p},\right. \\
& \left.\left(C_{m}+\sum_{i=1}^{n+2 p}\left(1-d_{i} / t_{\text {mini }}\right) C_{i}\right) /\left(1-\sum_{i=1}^{n+2 p} C_{i} / t_{\text {min } i}\right)\right\}
\end{aligned}
$$

and $C_{m}:=\max _{i} C_{i}$ is the maximum transmission time for all possible messages, $\lceil x\rceil^{+}=\min \left\{n \in \mathbb{N}_{0} \mid n \geq x\right\}$ and $\lfloor x\rfloor=$ $\max \{n \in \mathbb{Z} \mid n \leq x\}$, and $\phi_{s}=0$ for all sensor messages.

The previous set of equations assume that messages can be transmitted at any time. However, under IEEE 802.15.4 hard messages are not transmitted during the CAP (since guarantees cannot be provided) and during the inactive period (to save energy). We model this property by means of two dummy tasks with periods $t_{\min i}$ equal to the superframe duration S.D., and deadlines equal the inactive period (B.I. - S.D.) and $\triangle \mathrm{CAP}$ respectively. Moreover, the dummy task modeling the inactive period should have an offset equal to S.D. In this way, equations (13) and (14) can be used to analyze the schedulability under IEEE 802.15.4, where now $n$ represents the number of hard messages plus these two dummy messages. For other several related scheduling issues we refer the interested reader to [22].

As mentioned before, the schedulability analysis has to be based on the worst-case inter-transmission time $t_{\text {min }}$ as defined in (12) since the initial condition is in general not known in advance, or disturbances might steer the system to this worst-case condition. Nevertheless, the inherent dynamic nature of the self-triggered policy allows the scheduler to reallocate resources in an online manner. Different strategies could be applied for the dynamic bandwidth allocation: allocate those GTS to soft messages, or assign the GTS among all existing messages according to the needs of each node. For instance, a control system could take hold of GTS slots previously assigned to other control loops in order to improve its own performance.

\section{ExPERIMENTAL SETUP}

In order to evaluate the performance of the proposed approach, we built a scale version of an industrial setting where a wireless network is shared by two control loops and several independent nodes transmitting soft messages. The control loops are regulating two coupled water tank systems from Quanser [23], where the tanks are collocated with the sensors and actuators and communicate wirelessly with a controller node. The soft messages are monitoring messages with temperature, humidity and light values measured inside the room. Fig. 3 shows the setup of two water tank systems and the WSAN with seven independent monitoring nodes. This approach is very flexible and could be easily extended to the use of a large amount of sensor and actuator nodes under the same star network.

\section{A. Communication Network}

The wireless sensor platform chosen for this experiment is the Telos platform [18]. These nodes are equipped with a 250kbps 2.4 GHz Chipcon CC2420 IEEE 802.15.4 compliant
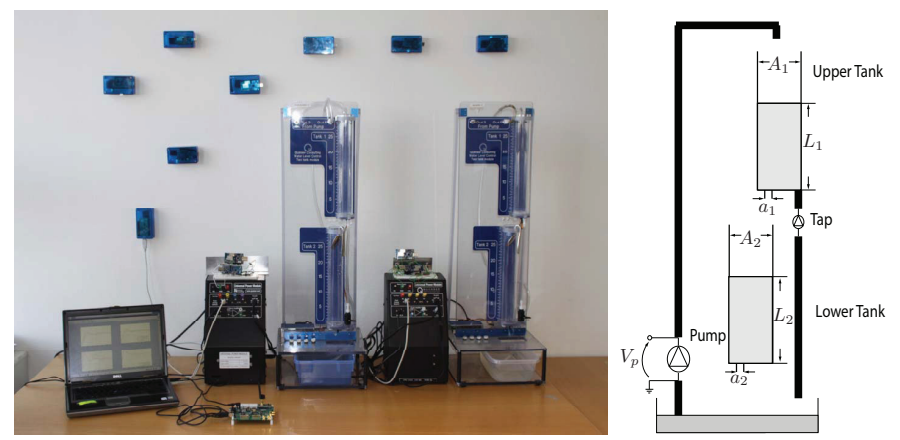

Fig. 3: WSAN setup with coupled water tank system and monitoring nodes. Block diagram of a water tank system.

radio and on-board sensors. Furthermore, this node has integrated Analog-to-Digital (ADC) and Digital-to-Analog (DAC) converters that allows us to use them as sensor and actuator nodes. The operating system used is TinyOS.

The IEEE 802.15.4 protocol has been partially implemented in TinyOS in TKN15.4 [24] and validated in the Telos platform. An extension of TKN15.4 to include the CFP and the GTS mechanism has been performed in [25] for the same platform. The implementation of the protocol used in our setup is based on [25] with the modification of the IEEE 802.15.4 standard as detailed in Sec. IV-A.

The MAC parameters design follows the considerations made in Sec. IV-B. We used a S.D. $=7680$ symbols = $117.2 \mathrm{~ms}$ and a B.I. $=61440$ symbols $=937.5 \mathrm{~ms}$. The inactive period has a total length of 53760 symbols $=814.6 \mathrm{~ms}$. Each of the 16 slots has a duration of $7.3 \mathrm{~ms}$. Additionally, we added the "sniffer" node CC2420 Development Kit from Texas Instruments IEEE 802.15.4 compliant, that allows for debugging and visualization of all the packets transmitted in the network. With this node we are able to properly evaluate our experimental setup and confirm the correct GTS scheduling, GTS times, acknowledgments and beacon messages.

\section{B. Coupled Water Tank System}

The Coupled Water Tank system by Quanser [23] consists of a pump, a water basin and two tanks of uniform cross sections. This system presents similar dynamical properties of an irrigation network of open water channels [2], where water flow is regulated by gates along the channels. Furthermore, the control of such a slow process resembles the control of HVAC systems [3]. The liquid in the lower tank flows to the water basin. A pump is responsible for pumping water from the water basin to the upper tank, which flows to the lower tank. The holes in each of the tanks have the same diameter. In order to introduce a disturbance in this system, the first tank has a drain tap such that, when opened, the liquid can flow directly to the water basin. The sensing of the water levels $L_{i}$ is performed by pressure sensors placed under each tank. The ratio between the sensor measurement and water level is given by $L_{i}=K_{s} \cdot V_{\text {out }}$, where $K_{s}=6.25 \mathrm{~cm} / \mathrm{V}$. The sensor signals are connected to the Quanser Universal Power Module (UPM) [23]. One 


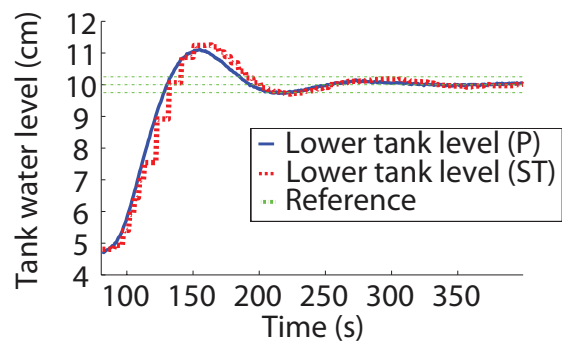

(a) Coupled Tank System 1

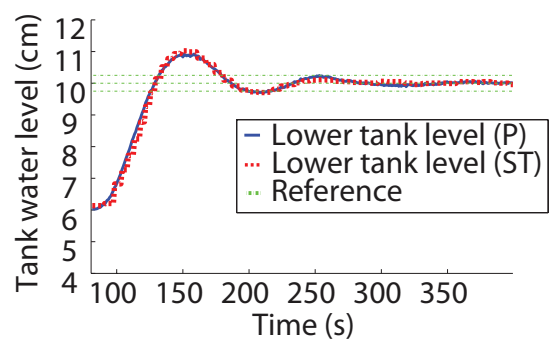

(b) Coupled Tank System 2

Fig. 4: Evolution of the water level $L_{2}$ of coupled tank systems 1 and 2, for ST and P strategies.

wireless sensor node interfaces the sensing channels of the UPM with an ADC, in order to sample the pressure sensor values for both tanks. The plant actuation is made through the pump motor which is actuated by the DAC of the wireless actuator node and the UPM. This voltage is fed to the UPM which actuates in the pump motor. The equations describing the dynamics of the two coupled water tanks are nonlinear. Thus, in order to apply the technique presented in Section III we linearized the dynamics around a quiescent point $L_{10}, L_{20}$, resulting in the linear dynamics:

$$
\begin{aligned}
& \Delta \dot{L}_{1}=-\frac{a_{1}}{A_{1}} \sqrt{\frac{g}{2 L_{10}}} \Delta L_{1}+\frac{K_{p}}{A_{1}} \Delta V_{p} \\
& \Delta \dot{L}_{2}=\frac{a_{1}}{A_{2}} \sqrt{\frac{g}{2 L_{10}}} \Delta L_{1}-\frac{a_{2}}{A_{2}} \sqrt{\frac{g}{2 L_{20}}} \Delta L_{2},
\end{aligned}
$$

where the states $\Delta L_{1}=L_{1}-L_{10}, \Delta L_{2}=L_{2}-L_{20}$ and $\Delta V_{p}=V_{p}-V_{p_{0}}$ represent the incremental values of the state and the input with respect to the quiescent point. In these equations $a_{i}$ is the outflow diameter of upper and lower tanks, $A_{i}$ is the diameter of the upper and lower tanks, $g$ is the gravitational acceleration in $\mathrm{cm} / \mathrm{s}^{2}, V_{p}$ is the voltage applied to the pump motor, $K_{p}$ is the pump motor constant, and $L_{i}$ is the height of the water in both upper and lower tanks. For this system we have the following parameters: $a_{1}=a_{2}=$ $0.178 \mathrm{~cm}^{2}, A_{1}=A_{2}=15.5 \mathrm{~cm}^{2}, K_{p}=2.775 \mathrm{~cm}^{3} / \mathrm{Vs}$ and $g=9.8 \mathrm{~m} / \mathrm{s}^{2}$. In equilibrium the value of the control input is $V_{p_{0}}=a_{1} \frac{\sqrt{2 g L_{10}}}{K_{p}}$ and $L_{10}=\frac{a_{2}^{2}}{a_{1}^{2}} L_{20}$.

Our objective is to achieve set-point tracking of the water level in the lower tank $L_{2}$ by adjusting the motor voltage $V_{p}$ accordingly. In order to ensure zero steady-state error we add an integral part to the state feedback controller. We perform this by adding an extra state $L_{I}$ satisfying $\frac{d}{d t} L_{I}=\Delta L_{2}-$
TABLE II: Wireless sensor of tank system 1: Number of transmissions, total current consumption over 320s of experimental validation and battery life expectation..

\begin{tabular}{|c|l|l|}
\hline & Periodic & Self-Triggered \\
\hline Number of transmissions & 427 & 62 \\
\hline Total current consumption & $0.0496 \mathrm{mAh}$ & $0.0293 \mathrm{mAh}$ \\
\hline Battery life (2900mAh) & 215.6 days & 365.5 days \\
\hline
\end{tabular}

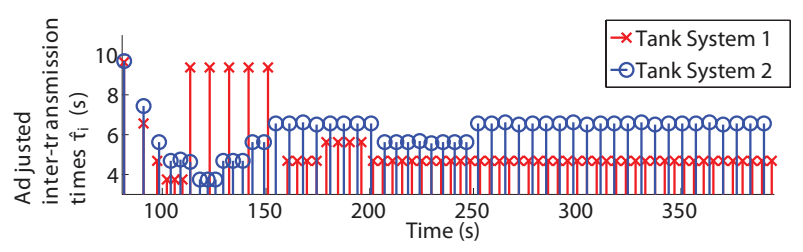

Fig. 5: Inter-transmission $\tau_{i}$ and adjust inter-transmission times $\hat{\tau}_{i}$ for the sensors on the coupled tank systems 1 and 2 when using the ST control strategy.

$\Delta L_{2 \text { ref }}$, where $\Delta L_{2 \text { ref }}$ is the desired final value for $\Delta L_{2}$. The stabilizing control input $\mathrm{u}=V_{p}$ is then defined as $\mathrm{u}=K \mathrm{x}$, where $\mathrm{x}=\left[\Delta L_{1}, \Delta L_{2}, L_{I}\right]$ and $K=[-0.16,-0.14,-0.019]$.

Each wireless sensor node will sample the pressure sensors $10 \mathrm{~ms}$ before transmitting the sensor value message to the PAN coordinator at a given GTS. In the following GTS the PAN coordinator will compute $\mathrm{u}=K \mathrm{x}$ and send this message to the wireless actuator node that applies voltage $u$ in the water pump. Upon the reception of the periodic beacon message, the wireless sensor node computes $L_{I}$ based on a forward Euler approximation of the solution of $\frac{d}{d t} L_{I}=\Delta L_{2}-\Delta L_{2 \text { ref. }}$.

The current consumption of each sensor is measured using the circuit in [19], where the output current-to-voltage is acquired by a real-time machine using Labview.

\section{GTS Scheduling Algorithm}

We define two different ranges of deadlines for the soft and the hard messages, to avoid having soft messages blocking hard messages. The scheduler computes the deadlines for the sensor and actuator messages according to (11). It is known that an upper bound on the self-triggered times $t_{i}$ needs to be imposed to guarantee robustness with respect to disturbances [15]. We fix that bound in $10 \mathrm{~s}$. The self-triggered implementation is determined by $R=0.1 Q$, where $\mathrm{Q}$ is selected as the identity matrix. We compute the minimum inter-transmission time for this system (as defined in (12)) using Lemma 4.1 in [15], which for this physical system gives us a minimum time of $1 \mathrm{~s}$. Hence the inter-transmission times $\tau_{i}$ for the control-related messages will be in the range $[1 s, 10 s]$. As mentioned in Sec. IV-B these times will be adjusted to $\hat{\tau}_{i}$ to be allocate at a GTS. Let us remark that by having a $\tau_{i}=1 \mathrm{~s}$ and a slot time of $7.3 \mathrm{~ms}$, following the current settings, we would be able to extend our setup to have a total of 68 coupled water tank systems under the coordination of a single PAN coordinator if there would be no maximum value of GTS slots in the IEEE 802.15.4 MAC protocol. By following the standard one is only able to perform the control of three coupled water tank systems in parallel, since the total number of messages 


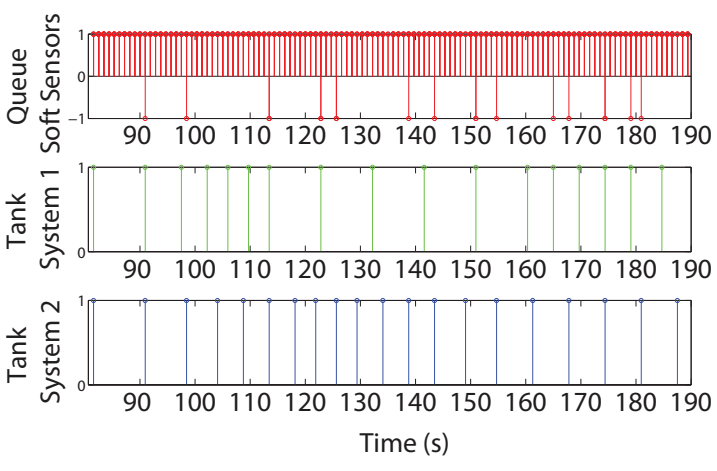

(a) Self-Triggered Controller

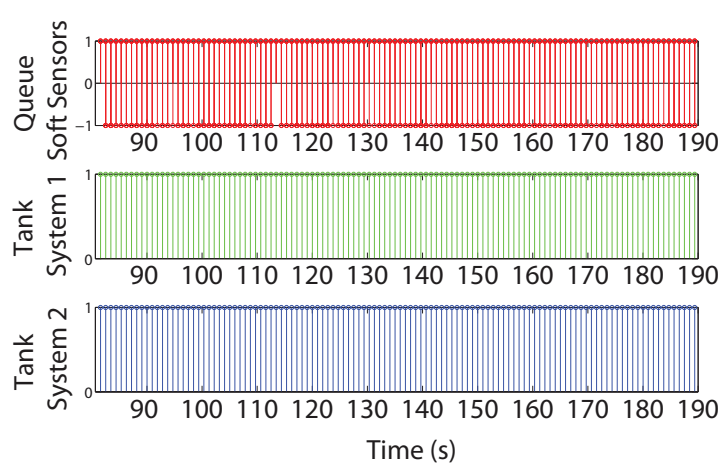

(b) Periodic Controller

Fig. 6: Scheduling and queue length of control tasks. Zoom in time between $[80,190] \mathrm{s}$.

to be schedule need to be below 7 . This provides a motivation for the extension of the maximum value of GTS slots allowed by the standard for control applications. The deadlines for the independent nodes will be randomly generated in the range $[1 \mathrm{~s}, 8 \mathrm{~s}]$. The schedule generated by the scheduler node is sent to the PAN coordinator and distributed to all the nodes in the following beacon message.

\section{Results}

First, we verified using the sniffer node, that with the proposed MAC mechanisms all the transmitted messages and beacon messages were properly received and achieving $100 \%$ reliability. Fig. 4 shows the time response of water tank systems 1 and 2 for self-triggered (ST) and periodic (P) control strategies. At time 100s a command is issued to raise the water level to $10 \mathrm{~cm}$. The objective of the control loops is then to use the pump to raise the water to the new set point starting from the previous set point of $4.8 \mathrm{~cm}$ and $6 \mathrm{~cm}$ for coupled tank system 1 and 2, respectively. The sampling period of the periodic implementation is set to $t_{\min }=1 \mathrm{~s}$, given by (12), since stability has to be guaranteed under all possible conditions. It is observed that both control implementations track the reference signal with similar behavior. The adjusted intertransmission times $\hat{\tau}_{i}$ of the self-triggered scheme are shown in Fig. 5. These times vary between $3.78 \mathrm{~s}$ and $9.37 \mathrm{~s}$, leading to a much lower number of transmissions than the periodic

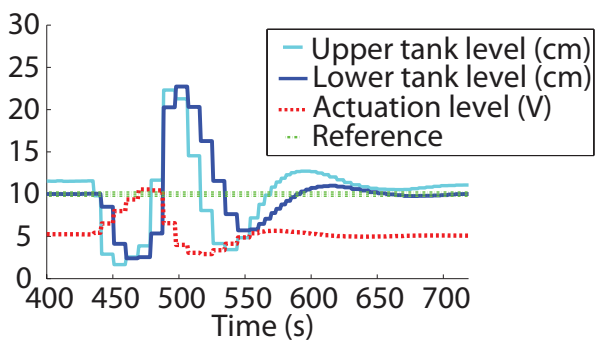

(a) Coupled Tank System 1

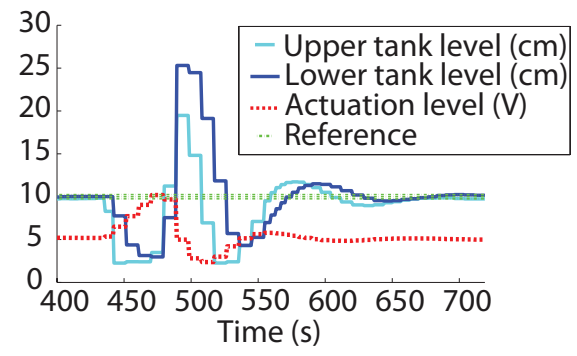

(b) Coupled Tank System 2

Fig. 7: Evolution of the water level $L_{2}$ of the coupled tank systems 1 and 2 and control inputs, for ST strategy when the tap is open between $t=[435,470] \mathrm{s}$.

scheme, but still achieving the same level of performance. We depict in Table II the values for the number of transmissions and total current consumption of the wireless sensor nodes reading the pressure sensors in the upper tank of both coupled tank systems. Even though the number of transmissions of the self-triggered control scheme is only $14.5 \%$ of the periodic, the battery lifetime increase is of $41 \%$ and not $85.5 \%$ as it could be expected ${ }^{2}$. This difference comes from the fact that the wireless nodes still need to wake up in order to receive the beacon message at each B.I., and therefore a reduction in the number of transmissions does not imply the same amount of energy savings. While the water tank systems are regulated, seven monitoring nodes are also transmitting soft messages to the PAN coordinator with information of the temperature, humidity and luminance in the room. Since they transmit low priority messages, these nodes enter a queue whenever their deadline expires. Since both control loops need 4 slots in total (sensing message and actuation message for both) there will be 3 slots available to be shared between the soft messages. Fig. 6 depict the queue status for the self-triggered and periodic cases from 100s to 200s. A value of 1 means that the soft message got delivered and a value of -1 if the deadline expired and the message enters the queue. It is clear that the self-triggered scheme allows for an overall higher network fairness and less queueing time for the soft messages by changing the intertransmission times in a dynamic fashion. This is achieved while guaranteeing control performance in the water tank systems.

\footnotetext{
${ }^{2}$ For battery lifetime expectation calculation, we sum the total current consumption of the wireless sensor over the 320 s period of reference tracking and repeat it until we consume the $2900 \mathrm{mAh}$ of battery capacity
} 
In order to confirm the robustness of our self-triggered control strategy we allow for level disturbance in both tank systems after the tracking of $10 \mathrm{~cm}$ in the lower tank is performed. Fig. 7 shows the case where the tap in the upper tank is opened at $t=435 \mathrm{~s}$ and closed at $t=470 \mathrm{~s}$, in both coupled tank systems. In this case the inter-sampling times varied between [1s,10s], allowing the self-triggered controller to successfully reject the disturbances applied to the water tank systems.

\section{CONCLUSION}

The present work provided an experimental illustration of the benefits of self-triggered control versus the traditional periodic paradigm, both in terms of energy savings and bandwidth allocation for WSANs applications. From a communication perspective, we proposed a modification of the IEEE 802.15.4 MAC which grants us great design flexibilities. As future work, we intend to study the benefits of other MAC designs that would increase performance on network resource usage, but also to look at the problem of performing self-triggered control over multi-hop networks. In this paper the controller was designed to guarantee stability and performance, but not to maximize the times between consecutive transmissions. Future work will focus on the implementation of minimum attention controllers [26], that minimize the amount of bandwidth required to stabilize a dynamical system.

\section{REFERENCES}

[1] A. Willig, "Recent and emerging topics in wireless industrial communications: A selection," IEEE Transactions on Industrial Informatics, vol. 4, no. 2, pp. $102-124$, May 2008.

[2] M. Cantoni, E. Weyer, Y. Li, S. Ooi, I. Mareels, and M. Ryan, "Control of large-scale irrigation networks," Proceedings of the IEEE, vol. 95, no. 1, pp. 75-91, 2007.

[3] T. Arampatzis, J. Lygeros, and S. Manesis, "A survey of applications of wireless sensors and wireless sensor networks," in Proceedings of the IEEE International Symposium on Intelligent Control, Mediterrean Conference on Control and Automation, 2005, pp. 719-724.

[4] C. Rozell and D. Johnson, "Power scheduling for wireless sensor and actuator networks," in Proceedings of the 6th international conference on Information processing in sensor networks, 2007, pp. 470-478.

[5] I. Akyildiz and I. Kasimoglu, "Wireless sensor and actuator networks: research challenges," Ad hoc networks, vol. 2, no. 4, pp. 351-367, 2004.

[6] J. Ploennigs, V. Vasyutynskyy, and K. Kabitzsch, "Comparative Study of Energy-Efficient Sampling Approaches for Wireless Control Networks," IEEE Transactions on Industrial Informatics, no. 99, p. 1, 2010.

[7] P. Antsaklis and J. Baillieul, "Technology of networked control systems," Proceedings of the IEEE, vol. 95, no. 1, 2007.

[8] K. Årzén, "A simple event-based PID controller," Preprints 14th World Congress of IFAC. Beijing, China, 1999.

[9] K. Åström and B. Bernhardsson, "Comparison of Riemann and Lebesgue sampling for first order stochastic systems," Proceedings of the 41st IEEE Conference on Decision and Control, vol. 2, 2002.

[10] P. Tabuada, "Event-triggered real-time scheduling of stabilizing control tasks," IEEE Transactions on Automatic Control, vol. 52(9), pp. 16801685, 2007.

[11] W. Heemels, J. Sandee, and P. van den Bosch, "Analysis of event-driven controllers for linear systems," Int. J. of Control, pp. 81(4), 571-590, 2008.

[12] M. Velasco, J. Fuertes, and P. Martí, "The self triggered task model for real-time control systems," 24th IEEE Real-Time Systems Symposium (work in progress), pp. 67-70, 2003.

[13] A. Anta and P. Tabuada, "To sample or not to sample: Self-triggered control for nonlinear systems," IEEE Transactions on Automatic Control, vol. 55, no. 9, pp. 2030-2042, 2010.
[14] X. Wang and M. Lemmon, "Self-triggered feedback control systems with finite-gain 12 stability," IEEE Transactions on Automatic Control, vol. 45, pp. 452-467, 2009.

[15] M. Mazo Jr, A. Anta, and P. Tabuada, "An ISS self-triggered implementation of linear controllers," Automatica, vol. 46, no. 8, pp. 1310-1314, 2010.

[16] A. Camacho, P. Martí, M. Velasco, C. Lozoya, R. Villa, J. Fuertes, and E. Griful, "Self-triggered networked control systems: An experimental case study," in IEEE International Conference on Industrial Technology (ICIT), 2010, pp. 123-128.

[17] IEEE 802.15.4 standard: Wireless Medium Access Control (MAC) and Physical Layer (PHY) Specifications for Low-Rate Wireless Personal Area Networks (WPANs), IEEE, 2006. [Online]. Available: http://www.ieee802.org/15/pub/TG4.html

[18] J. Polastre, R. Szewczyk, and D. Culler, "Telos: enabling ultra-low power wireless research," Information Processing in Sensor Networks 2005. Fourth International Symposium on, Apr. 2005.

[19] A. Prayati, C. Antonopoulos, T. Stoyanova, C. Koulamas, and G. Papadopoulos, "A modeling approach on the telosb wsn platform power consumption,” J. Syst. Softw., vol. 83, no. 8, pp. 1355-1363, 2010.

[20] G. Buttazzo, Hard real-time computing systems: predictable scheduling algorithms and applications. Springer-Verlag New York Inc, 2005.

[21] Q. Zheng and K. Shin, "On the ability of establishing real-time channels in point-to-point packet-switched networks," IEEE Transactions on Communications, vol. 42, no. 234, pp. 1096-1105, 1994.

[22] A. Anta and P. Tabuada, "On the benefits of relaxing the periodicity assumption for networked control systems over CAN," 30th IEEE RealTime Systems Symposium, pp. 3-12, 2009.

[23] Coupled Water Tanks, Quanser. [Online]. Available: http://www.quanser.com/english/downloads/products/Specialty/ CoupledTanks_PIS_031708.pdf

[24] J. Hauer, "TKNN15.4: An IEEE 802.15.4 MAC Implementation for TinyOS 2," TKN Technical Report Series, Telecommunication Networks Group, TU-Berlin, no. TKN-08-003, Mar 2009.

[25] A. Hernandez, "Wireless Process Control using IEEE 802.15.4 Protocol," Master Degree Thesis, Royal Institute of Technology, 2010. [Online]. Available: http://www.ee.kth.se/ araujo/hernandez10.pdf

[26] A. Anta and P. Tabuada, "On the minimum attention and anytime attention problems for nonlinear systems," 49th IEEE Conference on Decision and Control, 2010. 